\title{
Immunohistochemical profile of integrins in enlarged dental follicles and dentigerous cysts
}

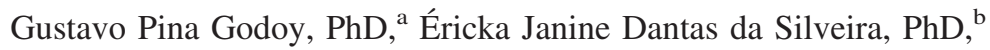 \\ Ruthinéia Diógenes Alves Uchoa Lins, $\mathrm{PhD},{ }^{\mathrm{a}}$ Lélia Batista de Souza, $\mathrm{PhD},{ }^{\mathrm{c}}$ \\ Roseana de Almeida Freitas, ${ }^{\mathrm{c}}$ and Lélia Maria Guedes Queiroz, $\mathrm{PhD},{ }^{\mathrm{c}}$ Campina Grande, Caicó, \\ and Natal, Brazil \\ STATE UNIVERSITY OF PARAÍBA, STATE'S UNIVERSITY OF RIO GRANDE DO NORTE, AND FEDERAL \\ UNIVERSITY OF RIO GRANDE DO NORTE
}

\begin{abstract}
Objective and study design. The morphological distinction between incipient dentigerous cyst (IDC) and enlarged pericoronal dental follicle (EDF) remains one of the most controversial questions in the literature. The objective of this study was to analyze the immunohistochemistry expression of alfa ${ }_{2}$ beta $_{1}$, alfa beta $_{1}$, and alfa $_{5}$ beta $_{1}$ in 23 cases of EDFs and 21 cases of IDCs.

Results. All integrins were immunopositive in the cases studied. A significant difference was detected regarding alfa $_{2}$ beta $_{1}$ integrin $(P<.0001)$ in which a higher expression was present in IDCs. Moreover, statistical difference was also found between basal and suprabasal cell layer in cystic epithelium $(P<.0034)$. The alfa beta $_{1}$ integrin expression showed significant difference $(P<.013)$ between EDF and IDC with a tendency of more pronounced staining in IDC. Conclusions. These results corroborate the possibility of histopathological distinction between EDF and IDC in which squamous metaplasia of reduced enamel epithelium to stratified epithelium would be the first event of cystic

transformation. (Oral Surg Oral Med Oral Pathol Oral Radiol Endod 2007;104:e29-e34)
\end{abstract}

The dental follicle (DF) or dental sac is an ectomesenchymal condensation that surrounds the crown of an unerupted tooth and is responsible for the orientation of tooth eruption. Its epithelial wall is formed by the reduced epithelium of the enamel organ, which consists of a single layer of cuboidal cells. ${ }^{1}$ This epithelium can give rise to some diseases such as odontogenic cysts and tumors, including dentigerous cysts. The dentigerous cyst consist of a developing follicular cyst that surrounds the crown of an unerupted tooth to which it adheres at the height of the cementoenamel junction. ${ }^{2}$

Controversies exist in the literature regarding the establishment of criteria for the conclusive diagnosis of incipient dentigerous cysts (IDC) or enlarged dental follicles (EDF). Some studies have indicated that a conclusive diagnosis of a dentigerous cyst can only be made based on the identification of a pathological cavity between the dental crown and ectomesenchymal portion during surgery, emphasizing that histopathological analysis is not able to distinguish between IDC and

\footnotetext{
aProfessor, State University of Paraíba.

'Professor, State's University of Rio Grande do Norte.

${ }^{\text {c} P r o f e s s o r, ~ O r a l ~ P a t h o l o g y, ~ F e d e r a l ~ U n i v e r s i t y ~ o f ~ R i o ~ G r a n d e ~ d o ~}$ Norte.

Received for publication Mar 6, 2007; returned for revision May 15, 2007; accepted for publication May 17, 2007.

$1079-2104 / \$$ - see front matter

(c) 2007 Mosby, Inc. All rights reserved.

doi:10.1016/j.tripleo.2007.05.024
}

EDF. ${ }^{3,4}$ Other investigators have emphasized that the distinction between these 2 entities can be made using criteria identified by the pathologist, which are mainly based on the type of epithelium present in the specimen, with the presence of a reduced enamel epithelium being indicative of DF epithelium. In the case of dentigerous cysts, squamous metaplasia of the reduced enamel epithelium appears to be the main feature, with the identification of a mainly continuous and thin epithelium in the specimen.,

Integrins are a family of heterodimers with an important function in different biological activities, mediating cell-cell and cell-extracellular matrix interactions. According to Decline and Rousselle ${ }^{7}$ and DeHart et al., ${ }^{8}$ integrins are involved in epithelial cell differentiation, a fact that indicates their use as additional differential markers in the distinction between IDC and EDF.

The objective of the present study was to compare the immunohistochemical expression of $\alpha_{2} \beta_{1}, \alpha_{3} \beta_{1}$, and $\alpha_{5} \beta_{1}$ integrins (ligands with higher affinity for collagen IV, laminin, and fibronectin, respectively) between IDC and EDF in order to provide additional data for the histopathological differentiation of these 2 entities.

\section{MATERIAL AND METHODS}

In the present descriptive study, the immunohistochemical expression of $\alpha_{2} \beta_{1}, \alpha_{3} \beta_{1}$, and $\alpha_{5} \beta_{1}$ integrins was analyzed in 21 cases of IDC and 23 cases of EDF, 
Table I. Antibodies used

\begin{tabular}{|c|c|c|c|c|}
\hline Specificity & Clone & Antigenic retrieval & Dilution & Incubation time \\
\hline$\alpha 2 \beta 1$ integrin* & $\mathrm{BHA}_{2,1}$ & Pepsin $0.5 \%\left(37^{\circ} \mathrm{C} / 30 \mathrm{~min}\right)$ & $1 / 1000$ & $60 \mathrm{~min}$ \\
\hline$\alpha 3 \beta 1$ integrin & $\mathrm{M}-\mathrm{KD}_{102}$ & Pepsin $0.5 \%\left(37^{\circ} \mathrm{C} / 30 \mathrm{~min}\right)$ & $1 / 500$ & $60 \mathrm{~min}$ \\
\hline$\alpha 5 \beta 1$ integrin & $\mathrm{FBS}_{5}$ & Pepsin $0.5 \%\left(37^{\circ} \mathrm{C} / 30 \mathrm{~min}\right)$ & $1 / 1000$ & $60 \mathrm{~min}$ \\
\hline
\end{tabular}

*Integrins were from Chemicon International, Temeluca, CA.

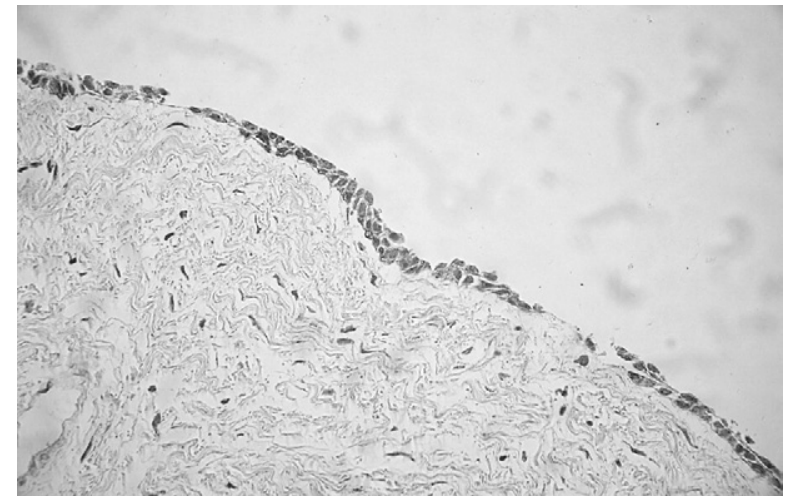

Fig. 1. Diffuse and granular distribution of $\alpha_{2} \beta_{1}$ integrin at intercellular contacts in the epithelial-connective tissue interface in $\mathrm{EDF}(\mathrm{SABC}, \times 200)$.

using as an inclusion criterion the diagnostic hypothesis of dentigerous cyst or DF as stated in the clinical chart sent by the dental surgeon for morphological examination. The surgeon had this doubt when in the radiographic examination the space between the crown and the border of the pericoronal radiolucency did not exceed $2.5 \mathrm{~mm}$. The following histopathological inclusion criteria were applied: for dentigerous cysts, the observation of a thin stratified squamous epithelial lining the cystic cavity, exhibiting areas of continuity and arranged throughout a connective capsule of variable density; for DF, observation of a thin and discontinuous simple cuboidal reduced enamel epithelium, in addition to loose fibrous connective tissue.

The paraffin-embedded material was cut into $3-\mu \mathrm{m}$ thick sections and the specimens were mounted on previously cleaned glass slides with organosilane (3aminopropyltriethoxy-silano [Sigma Chemical Co, St Louis, MO]) as adhesive. The specimens were analyzed by a streptavidin-biotin immunohistochemical method (SABC, streptavidin biotin complex) using the catalyzed signal amplification system and anti-integrin $\alpha_{2} \beta_{1}, \alpha_{3} \beta_{1}$, and $\alpha_{5} \beta_{1}$ antibodies (Table I).

Immunostained cells were analyzed regarding the presence or absence, intensity, and pattern of distribution of staining. Labeling intensity was scored from 0 to 2 , where $0=$ absent, $1=$ discrete, and $2=$ intense labeling. This intensity was evaluated in a general manner in the specimen, and also at specific sites in-

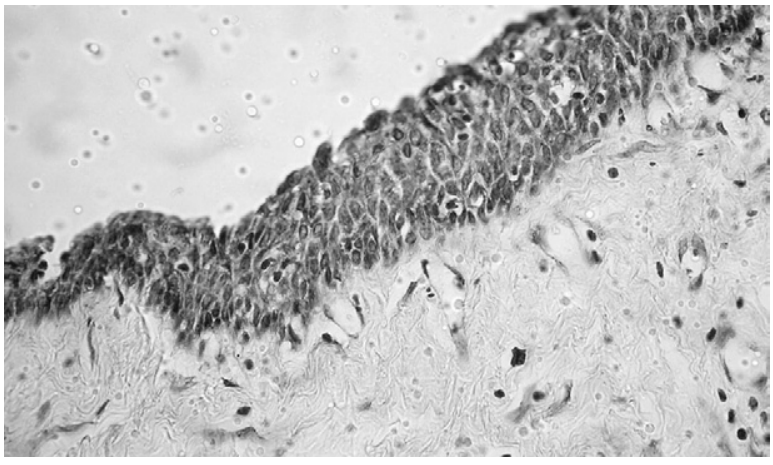

Fig. 2. Diffuse and granular distribution of $\alpha_{2} \beta_{1}$ integrin in IDC $($ SABC, $\times 200)$.

cluding the epithelial-connective tissue interface, intercellular contacts, and islands of odontogenic epithelium. The discrete labeling corresponded to that where visual verification was present but barely evident for the tonality of the coloration, and the opposite was considered for the intense labeling. In IDC, the intercellular contacts were evaluated separately in the basal and suprabasal layers because of the presence of stratification in this epithelium, in contrast to DF where the epithelium consists of a single cell layer. With respect to the pattern of distribution, focal staining was defined when detected in groups of cells irrespective of their number, whereas a diffuse pattern was established in the case of homogeneous cell labeling throughout the specimen. The samples were analyzed by a single observer under a Leica ATC 2000 light microscope (Wetzlar, Germany) at $\times 400$ magnification at 2 different time points.

The study was approved by the Research Ethics Committee of the Federal University of Rio Grande do Norte.

\section{RESULTS}

All cases analyzed were immunopositive for $\alpha_{2} \beta_{1}$, $\alpha_{3} \beta_{1}$, and $\alpha_{5} \beta_{1}$ integrins; however, some peculiarities were observed for each integrin. Discrete staining for $\alpha_{2} \beta_{1}$ integrin was observed in 20 (86.96\%) EDF cases, showing a diffuse distribution at intercellular contacts and at the epithelial-connective tissue interface in all cases (Fig. 1). Eighteen of the EDF cases studied pre- 


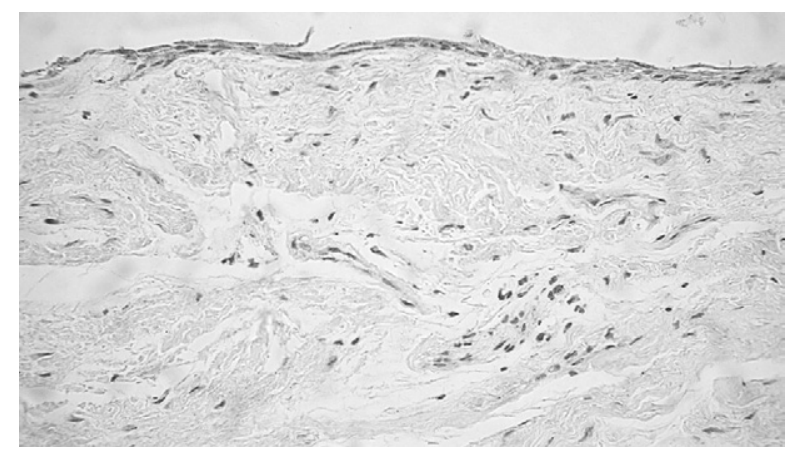

Fig. 3. Discrete staining of $\alpha_{3} \beta_{1}$ integrin in EDF (SABC, $\times 200)$.

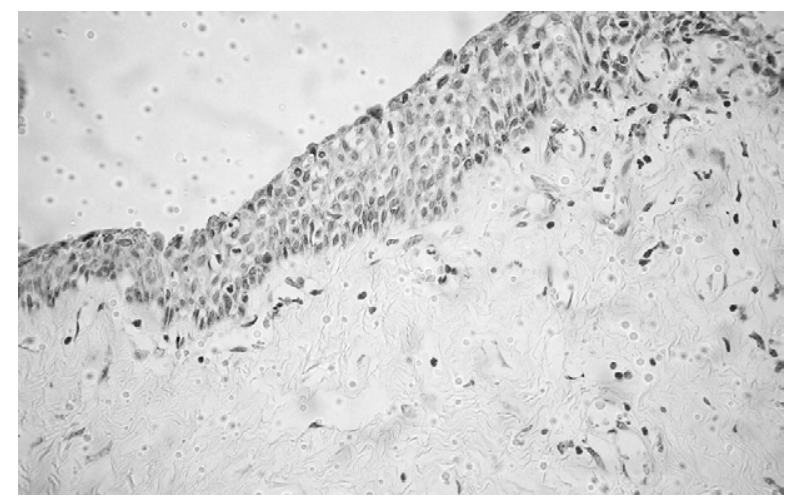

Fig. 4. Immunoexpression discret for $\alpha_{3} \beta_{1}$ integrin with a diffuse pattern of distribution and granular aspect at intercellular contacts and at the epithelial-connective tissue interface in IDC $(\mathrm{SABC}, \times 200)$.

sented islands of odontogenic epithelium scattered throughout the specimen, with $15(83.33 \%)$ of them showing discrete labeling for $\alpha_{2} \beta_{1}$, whereas intense labeling was observed in $3(16.67 \%)$.

In contrast, in IDC $\alpha_{2} \beta_{1}$ integrin exhibited intense labeling in $14(66.67 \%)$ of 21 cases, with a diffuse distribution similar to that noted in DF being observed in all cases (Fig. 2). In addition, labeling tended to be more intense in basal cells of the epithelial wall compared to suprabasal cells. Only 8 (38.10\%) IDC cases presented islands of odontogenic epithelium where staining for this integrin was discrete.

With respect to $\alpha_{3} \beta_{1}$ integrin, discrete staining was observed in most EDF cases (86.96\%), showing a predominantly focal distribution (82.6\%) and epithelial cells with granular cytoplasm (Fig. 3). Islands of odontogenic epithelium were identified in 17 cases, 15 of them (88.24\%) showing discrete staining for this integrin.

In IDC, expression of $\alpha_{3} \beta_{1}$ integrin was discrete in 11 cases $(52.28 \%)$, showing a diffuse pattern in all cases, both at intercellular contacts and at the epithelial-

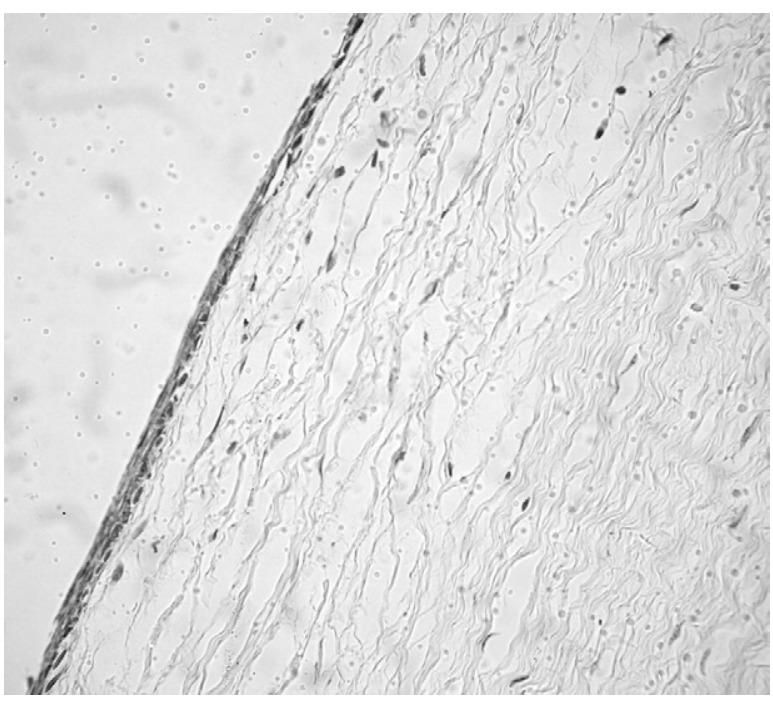

Fig. 5. Intense staining for $\alpha_{5} \beta_{1}$ integrin was in EDF (SABC, $\times 200)$.

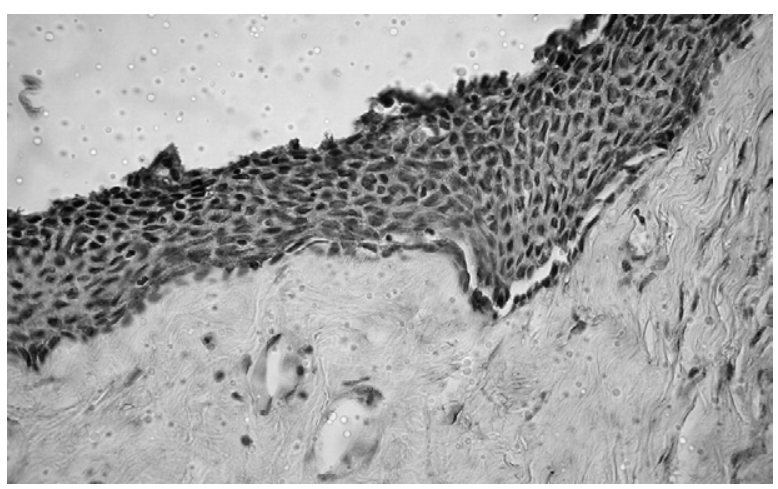

Fig. 6. Intense labeling for $\alpha_{5} \beta_{1}$ with a diffuse and granular distribution pattern in IDC $($ SABC, $\times 200)$.

connective tissue interface (Fig. 4). No difference in the expression of $\alpha_{3} \beta_{1}$ integrin was observed between basal and suprabasal cells of the epithelial wall. Islands of odontogenic epithelium were observed in only 8 $(38.10 \%)$ of the 21 cases, with $6(75 \%)$ of them presenting discrete staining and $2(25 \%)$ presenting intense staining for this integrin. There was a significant difference in the labeling pattern when comparing EDF and IDC $(P<.0001)$.

Staining for $\alpha_{5} \beta_{1}$ was intense in 20 (86.96\%) of the EDF cases analyzed (Fig. 5), with the distribution pattern being diffuse in all cases, both at intercellular contacts and at the epithelial-connective tissue interface. Islands of odontogenic epithelium were observed in $16(69.57 \%)$ cases; $14(87.5 \%)$ of them showing intense labeling. 
Intense labeling for $\alpha_{5} \beta_{1}$ was observed in 18 $(85.72 \%)$ of the IDC cases (Fig. 6). In all cases, the distribution pattern was diffuse and no difference in the expression pattern was observed between basal and suprabasal cells of the epithelial border. In addition, islands of odontogenic epithelium were identified in 12 $(57.14 \%)$ cases, $10(83.33 \%)$ showing intense labeling for integrin $\alpha_{5} \beta_{1}$.

Comparison of the overall expression of $\alpha_{2} \beta_{1}, \alpha_{3} \beta_{1}$, and $\alpha_{5} \beta_{1}$ integrins between EDF and IDC revealed a significant difference for $\alpha_{2} \beta_{1}(P=.0001)$ and $\alpha_{3} \beta_{1}$ $(P=.013)$, while no difference was observed for $\alpha_{5} \beta_{1}$ integrin $(P>.05)$. The expression of ta2b1 and a3b1 integrins had always presented a trend to be more intense in IDC than in EDF. Furthermore, separate comparison by Dunn's multiple comparison post-test showed a significant difference between the expression pattern of these integrins for each entity. For EDF, a significant difference was observed between $\alpha_{2} \beta_{1}$ and $\alpha_{5} \beta_{1}$ integrins and between $\alpha_{3} \beta_{1}$ and $\alpha_{5} \beta_{1}$ integrins. In contrast, in IDC a significant difference was observed when comparing the results between all integrins, i.e., between $\alpha_{2} \beta_{1}$ and $\alpha_{3} \beta_{1}$, between $\alpha_{2} \beta_{1}$ and $\alpha_{5} \beta_{1}$, and between $\alpha_{3} \beta_{1}$ and $\alpha_{5} \beta_{1}$.

The method used for the determination of the intensity of expression of $\alpha_{2} \beta_{1}, \alpha_{3} \beta_{1}$, and $\alpha_{5} \beta_{1}$ integrins at the intercellular contacts was different for each entity. In the case of EDF, the intensity of expression is reported as a single value because of the presence of a single epithelial cell layer and was similar to the overall expression of integrins in this epithelium. In contrast, in IDC the labeling intensity at the intercellular contacts was subdivided into 2 groups: cells of the basal layer and cells of the suprabasal layer. We opted for this type of assessment because of the stratification of the epithelium in IDC. In the case of $\alpha_{2} \beta_{1}$ integrin, there was a significant difference in expression between basal and suprabasal cells $(P=.0034)$, while no difference was observed for $\alpha_{3} \beta_{1}$ or $\alpha_{5} \beta_{1}$ integrin $(P>.05)$.

\section{DISCUSSION}

In view of the controversies that exist in the literature regarding the histopathological distinction between EDF and IDC, the present study compared the immunohistochemical expression of $\alpha_{2} \beta_{1}, \alpha_{3} \beta_{1}$, and $\alpha_{5} \beta_{1}$ integrins between EDF and IDC to determine whether the expression of these integrins may help distinguish between these 2 entities.

In the present study, all cases of DF and IDC were positive for the integrins analyzed, showing a granular expression pattern preferentially located in the cytoplasm and, in most cases, close to the cytoplasmic cell membrane. This type of pericellular expression has also been identified by Jensen and Wheelock ${ }^{9}$ in epidermal keratinocytes, supporting the view of the importance of integrins in cell-cell and cell-extracellular matrix adhesion through the basement membrane. Modolo et al. ${ }^{10}$ also observed positive staining for integrins in dental germ, dental lamina, and oral mucosal epithelium, demonstrating the importance of integrins for the architectural maintenance of different types of epithelium.

In the present study, the staining pattern for $\alpha_{2} \beta_{1}$ integrin differed between DF and IDC, with this staining being more discrete in DF both in cells of the reduced enamel epithelium and in islands of odontogenic epithelium if present in the specimen. In contrast, predominantly intense staining was observed in IDC, especially in cells of the basal layer of the cystic epithelial wall. These findings agree with Bennett et al., ${ }^{11}$ who reported that the $\alpha_{2}$ subunit is expressed in larger amounts in proliferating cells. This fact explains the tendency toward a more significant expression in basal cells of the cystic epithelium since, although not a marked feature of this epithelium, these cells show constant proliferative activity in the epithelial wall, in contrast to cells of the reduced enamel epithelium, which tend to be quiescent. This fact was confirmed in the present study by the demonstration of a significant difference in the expression of $\alpha_{2} \beta_{1}$ integrin at the epithelial-connective tissue interface between DF and IDC $(P<.0001)$.

Nishimura et al. ${ }^{12}$ reported that the integrin $\alpha_{2}$ subunit plays an effective role in the stratification of squamous epithelia, including the epithelium of cystic lesions. The authors observed that $\alpha_{2}$ integrin was more expressed on basal cells of the epithelial wall of dentigerous cysts and odontogenic keratocysts, and suggested that the reduction of expression in subsequent layers is a result of a decrease in cell-cell adhesion and favors the desquamation of more superficial cells. Similar findings were obtained in the present investigation showing a significantly more intense expression of $\alpha_{2} \beta_{1}$ integrin in basal cells compared with suprabasal cells in the epithelium of IDC.

The identification of $\alpha_{3} \beta_{1}$ integrin in all cases analyzed in the present study agrees with the findings of DeHart et al., ${ }^{8}$ who pointed out the importance of the interaction between $\alpha_{3} \beta_{1}$ integrin and laminin-5 in basement membranes, which favors adhesion of epithelial cells to this membrane as well as cell-cell adhesion, with the consequent organization and remodeling of different types of epithelial cells. Comparison of the overall labeling intensity of $\alpha_{3} \beta_{1}$ integrin between DF and IDC revealed a significant difference between these 2 entities despite the predominantly discrete staining pattern in the 2 groups. This finding agrees with Nishimura et al. ${ }^{12}$ who emphasized the importance of $\alpha_{3} \beta_{1}$ integrin for the stratification of cystic epithelia analyzed 
in that study, a fact explaining the tendency toward higher expression of $\alpha_{3} \beta_{1}$ integrin in IDC compared to DF. The same was demonstrated in the present study by the significant difference in $\alpha_{3} \beta_{1}$ expression at the epithelial-connective tissue interface between DF and IDC.

Hemler and Rutishauser ${ }^{13}$ and Dyce et al. ${ }^{14}$ reported the production and activation of some metalloproteinases mediated by $\alpha_{3} \beta_{1}$ integrin, a process that would also explain the more intense expression of this integrin in IDC compared to DF in view of the importance of some metalloproteinases in bone matrix degradation and consequent cystic expansion.

A significant difference was observed in the distribution pattern of $\alpha_{3} \beta_{1}$ integrin between DF and IDC, with the pattern being predominantly focal in the first group and diffuse in the second group in all cases. This finding can be explained by the reports of DeHart et al. ${ }^{8}$ They demonstrated the importance of $\alpha_{3} \beta_{1}$ integrin in the structural organization of pavement epithelia such as that found in IDC in the present study, in which this integrin is necessary for cell-cell and cell-extracellular matrix adhesion, therefore exhibiting a diffuse staining pattern in these lesions. On the other hand, the weak adhesion of reduced enamel epithelial cells to underlying connective tissue in DF reported by Stanley et al. ${ }^{15}$ may be a possible explanation for the focal staining observed for $\alpha_{3} \beta_{1}$ integrin in these lesions. This fact explains the observation that this epithelium was absent or showed large areas of discontinuity in DF specimens since studies by Hemler and Rutishauser ${ }^{13}$ and Darribere and Schwarzbauer ${ }^{16}$ have demonstrated the importance of binding of $\alpha_{3} \beta_{1}$ integrin to laminin-5 for the organization of epithelial cells in the basement membrane.

According to $\mathrm{Su}$ et al. ${ }^{17}, \alpha_{5} \beta_{1}$ integrin plays an important role in cellular differentiation, a fact confirmed in the present study showing intense staining in cells of the reduced enamel epithelium of DF and in cells of the stratified pavement epithelium of IDC. These cells are characterized by well-established differentiation, a fact explaining the high expression of $\alpha_{5} \beta_{1}$ integrin. However, these results disagree with the findings of Kosmehl et al., ${ }^{18}$ who observed discontinuous staining for $\alpha_{5} \beta_{1}$ integrin at the epithelial-basement membrane interface in normal oral mucosa and a more intense staining in poorly differentiated epidermoid carcinoma. According to these authors, expression of $\alpha_{5} \beta_{1}$ integrin tended to be higher in more undifferentiated cells.

According to Labat-Robert, ${ }^{19}$ the expression of $\alpha_{5} \beta_{1}$ integrin inactivates genes responsible for cell proliferation, thus favoring the control of tissue growth. This fact might explain the intense expression of $\alpha_{5} \beta_{1}$ inte- grin both in the reduced enamel epithelium of DF and in the stratified pavement epithelium of dentigerous cysts, even at the epithelial-connective tissue interface where no significant difference in the intensity of expression between DF and IDC was observed. These findings support the importance of the interaction between $\alpha_{5} \beta_{1}$ integrin and fibronectin, a protein abundantly present in the underlying basement membrane, for the control of cell proliferation.

The results regarding the expression of $\alpha_{2} \beta_{1}, \alpha_{3} \beta_{1}$, and $\alpha_{5} \beta_{1}$ integrins in DF and IDC suggest that squamous metaplasia is indeed the first visible sign of cystic transformation of DF, since significant differences in the integrin expression pattern were observed between the 2 entities. These findings agree with Eisenberg, ${ }^{20}$ who reported the possibility of histopathological distinction between DF and IDC. However, caution should be taken before a conclusive diagnosis is made, with clinical and surgical information being highly important. In this respect, Katchburian and Arana ${ }^{21}$ reported that cells of the reduced enamel epithelium fuse with basal cells of the oral mucosal epithelium during the normal process of tooth eruption, a fact that might confuse the pathologist when identifying areas of stratified pavement epithelium in semi-impacted DF specimens. Therefore, the criteria reported for the distinction between DF and IDC are only valid for fully impacted teeth.

\section{REFERENCES}

1. Miller CS, Bean L. Pericoronal radiolucencies with and without radiopacities. Dent Clin North Am 1994;38:51-61.

2. Kramer IRH, Pindborg JJ, Shear M. The WHO histologic typing of odontogenic tumours: a commentary of the second edition. Cancer 1992;70:2988-94.

3. Daley TD, Wysocki GP. New developments in selected cysts of the jaws. J Can Dent Assoc 1997;63:526-32.

4. Damante JH, Fleury RN. A contribution to the diagnosis of the small dentigerous cyst or the paradental cyst. Bras Oral Res 2001;15:238-46.

5. Glosser JW, Campbell JH. Pathologic change in soft tissues associated with radiographically "normal" third molar impactions. Br J Oral Maxillofac Surg 1999;37:259-60.

6. Adelsperger J, Campbell JH, Coates DB, Summerlin DJ, Tomich CE. Early soft tissue pathosis associated with impacted third molars without pericoronal radiolucency. Oral Surg Oral Med Oral Pathol Oral Radiol Endod 2000;89:402-6.

7. Decline F, Rousselle P. Keratinocyte migration requires $\alpha_{2} \beta_{1}$ integrin-mediated interaction with the laminin-5 $\gamma 2$ chain. J Cell Sci 2001;114:811-23.

8. DeHart GW, Healy KE, Jones JCR. The role of $\alpha_{3} \beta_{1}$ integrin in determining the supramolecular organization of laminin-5 in the extracellular matrix of keratinocytes. Exp Cell Res 2003;283: 67-79.

9. Jensen PJ, Wheelock MJ. $\beta_{1}$ integrins do not have a major role in keratinocyte intercellular adhesion. Exp Cell Res 1995;219: 322-31. 
10. Modolo F, Martins MT, Loducca SV, de Araujo VC. Expression of integrin subunits $\alpha_{2}, \alpha_{3}, \alpha_{5}, \alpha_{v}, \beta_{1}, \beta_{3}$ and $\beta_{4}$ in different histological types of ameloblastoma compared with dental germ, dental lamina and adult lining epithelium. Oral Dis 2004;10: 277-82.

11. Bennett JH, Carter DH, Alavi AL, Beresford JN, Walsh S. Patterns of integrin expression in a human mandibular explant model of osteoblast differentiation. Arch Oral Biol 2001;46:229-38.

12. Nishimura A, Ueno S, Niwa S, Osugi Y, Shinoda Y, Mushimoto $\mathrm{K}$, et al. Correlation of lining thickness and expression of $\alpha 2$ and $\alpha 3$ integrins within the epithelial lining of odontogenic cysts. J Osaka Dent Univ 1998;23:43-6.

13. Hemler ME, Rutishauser U. Cell-to-cell contact and extracellular matrix. Curr Opinion Cell Biol 2000;12:539-41.

14. Dyce OH, Ziober AF, Weber RS, Miyazaki K, Khariwala SS, Feldman M, et al. Integrins in head and neck squamous cell carcinoma invasion. Laryngoscope 2002;112:2025-32.

15. Stanley HR, Krogh H, Pannkuk E. Age changes in the epithelial components of the follicles (dental sacs) associated with impacted third molars. Oral Surg Oral Med Oral Pathol 1965; 129:128-139.

16. Darribere T, Schwarzbauer JE. Integrins: regulators of embryogenesis. Biol Cell 2000;92:5-25.
17. Su JM, Gui L, Zhou YP, Zha XL. Expression of focal adhesion kinase and $\alpha_{5}$ and $\beta_{1}$ integrins in carcinomas and its clinical significance. World J Gastroenterol 2002;8:613-8.

18. Kosmehl H, Berndt A, Katenkamp D, Hyckel P, Stiller KJ, Gabler $\mathrm{U}$, et al. Integrin receptors and their relationship to cellular proliferation and differentiation of oral squamous cell carcinoma. A quantitative immunohistochemical study. J Oral Pathol Med 1995;24:343-8.

19. Labat-Robert J. Fibronectin in malignancy. Effect of aging. Cancer Biol 2002;12:187-95.

20. Eisenberg E. Dental follicular tissue: misinterpretation as odontogenic tumors [discussion]. J Oral Maxillofac Surg 1993;51:767-8.

21. Katchburian E, Arana V. Histologia e Embriologia Oral. 1st ed. São Paulo, Brazil: Ed. Medicina Panamericana; 1999.

\section{Reprint requests:}

Gustavo Pina Godoy, PhD

Universidade Federal do Rio Grande do Norte

Departamento de Odontologia

Programa de Pós-Graduação em Patologia Oral Av. Senador Salgado Filho, 1787, Lagoa Nova

Natal-RN Brasil CEP: 59056-000

gruiga@hotmail.com 